\title{
A Robust Folk Theorem for the Prisoner's Dilemma *
}

\author{
Jeffrey C. Ely ${ }^{\dagger}$ Juuso Välimäki ${ }^{\ddagger}$
}

December 23, 1999

\begin{abstract}
We prove the folk theorem for the Prisoner's dilemma using strategies that are robust to private monitoring. From this follows a limit folk theorem: when players are patient and monitoring is sufficiently accurate, (but private and possibly independent) any feasible individually rational payoff can be obtained in sequential equilibrium. The strategies used can be implemented by finite (randomizing) automata.
\end{abstract}

\footnotetext{
${ }^{*}$ Thanks to Görkem Celik for valuable research assistance.

${ }^{\dagger}$ Economics Department, Northwestern University. ely@nwu.edu

${ }^{\ddagger}$ Economics Department, University of Southamption. valimaki@soton.ac.uk
} 
The folk theorem for discounted repeated games states that every payoff vector that is feasible and individually rational is an equilibrium payoff when players are sufficiently patient. A proof of the folk theorem first appeared in Fudenberg and Maskin (1986) for subgame-perfect equilibria of repeated games with perfect monitoring. Perfect monitoring means that the history of chosen actions is always common knowledge among the players. In many important economic applications, players monitor one another imperfectly: each observes a noisy signal of the actions chosen by others. Thus, starting with the early papers by Radner (1985) and Green and Porter (1984), attention turned to repeated games with imperfect monitoring. The early applications were to situations in which monitoring, while imperfect, was public: the random payoff-relevant outcome in each stage was assumed common knowledge among the players. Exploiting the structure of public monitoring, dynamic programming techniques can be used to characterize the set of perfect public equilibrium payoffs as in Abreu, Pearce, and Stachetti (1986) and Abreu, Pearce, and Stachetti (1990). This approach to imperfect monitoring culminated in the folk theorem of Fudenberg, Levine, and Maskin (1994) which identified conditions on the public monitoring technology which ensured that all feasible and individually rational payoffs could be supported in equilibrium.

In repeated games with monitoring by privately observed signals, these techniques do not apply and whether the folk theorem extends is still an open question. Indeed, for some games, whether there are any sequential equilibria different from repetition of stage-game Nash profiles is unresolved. ${ }^{1}$ Numerous negative results emphasize the difficulties invovled. Matsushima (1991) considers repeated play of stage games with a unique Nash equilibrium and monitoring by conditionally independent private signals. Conditional independence means that for each action profile, the players' private signals are distributed independently of one another. Each individual's private signal may be arbitrarily informative about the realized action profile, but conditional on that action profile, each player's signal is uninformative about the signals observed by other players. Matsushima shows that if there is any pure-strategy equilibrium different from repetition of the stage game Nash profile, it must involve conditioning on payoff-irrelevant history. Specifically, there must be a player $i$ and a pair of histories for $i$ which give rise to identical

\footnotetext{
${ }^{1}$ For a particularly problematic example, see the discussion in Mailath and Morris (1998) of the "convention game" originally studied by Shin and Williamson (1991)
} 
beliefs over the opponents' continuation strategies but which nevertheless induce distinct continuation play by $i$. In particular, this implies that equilibria cannot be "strict." 2

Many of the strategies used to prove folk theorems in environments with public monitoring fail to be even approximate equilibria when monitoring is at all imperfect but conditionally independent. For example, consider the strategies used by Fudenberg and Maskin (1991) to prove the folk theorem under perfect monitoring. These strategies begin in a cooperative phase in which players play a deterministic sequence of pure action profiles until some player deviates from that sequence. Such a deviation triggers a punishment in which the deviating player is minmaxed. Continuation strategies are constructed so that the non-deviating players have an incentive to carry out the punishment and return to the cooperative phase. When the discount factor is close enough to one, each player has a strict incentive to follow his equilibrium strategy after every history.

It is this strictness property that implies that even the slightest conditinally independent private monitoring imperfections destroy the equilibrium. To see this, consider a player $i$ who has detected a deviation by player $j$ in stage 1 . Since $i$ knows that $j$ is following his equilibrium strategy, $i$ knows that $j$ has not deviated and in fact that no player has deviated. Since the equilibrium was constructed so that $i$ has a strict incentive to cooperate after histories in which no player has deviated, $i$ will not punish but will instead continue to cooperate. But this unwillingness to punish signals of bad behavior eliminates the necessary incentives to cooperate in the first place.

This naturally raises the question of what equilibria of games with perfect monitoring are robust to private monitoring imperfections. That is, for which (if any) equilibria is there guaranteed to be a nearby equilibrium when monitoring is nearly perfect, but private. In this paper, we focus on the repeated prisoners' dilemma and prove a robust folk theorem: for sufficiently patient players, every feasible, individually rational payoff can be achieved by equilibrium strategies that are robust to private monitoring. This implies a limit folk-theorem: For monitoring technologies sufficiently accurate and discount factors close enough to one, any individually rational payoff can be

\footnotetext{
${ }^{2}$ Bhaskar (1998) derives an even stronger necessary condition from a model in which each player's payoffs are randomly perturbed in each period independently of history and are private information to that player. In a repeated game with overlapping generations of players (hence private monitoring), his condition implies that any equilibrium must be a repetition of stage-game Nash outcomes.
} 
achieved in a sequential equilibrium of the private monitoring game.

Related results have been obtained elsewhere. Sekiguchi (1997) was the first to show that the mutual cooperation payoff can be achieved in a class of prisoners' dilemma games when monitoring is nearly perfect. ${ }^{3}$ Mailath and Morris (1998) show that some trigger strategies are robust to monitoring imperfections that are approximately "public." Monitoring is approximately public when a players realized signal is informative of the signals observed by others (even after conditioning on the realized action.) Conditional independence is therefore ruled out.

To prove that trigger-strategy and other strict equilibria are robust, the problem of discontinuous conditional beliefs must be circumvented. Conditional beliefs about opponents' continuation strategies after histories involving a deviation change discontinuously when slight monitoring imperfections are introduced. This can be seen in the above discussion of the Fudenberg and Maskin (1991) strategies. When monitoring is perfect, when player $i$ observes a deviation in stage 1 , he is certain that his opponents will move into the punishment phase. But when monitoring is conditionally independent, following the corresponding (private) history player $i$ is nearly certain that his opponents will continue to cooperate. This observation, together with the Matsushima result, make it clear that strict equilibria cannot be robust to all private monitoring imperfections.

Mailath and Morris (1998) restrict attention to monitoring technologies in which there is sufficient correlation in the players' private signals, i.e. nearly public monitoring. When monitoring is approximately public, player $i$ will be nearly certain that the opponents have also observed a deviation and will begin punishing, even though it is common knowledge among the players that no deviation has occured. Notice that conditional beliefs are no longer discontinuous once the monitoring imperfections are restricted in this way. ${ }^{4}$

Our approach to the folk theorem deals with discontinuous conditional beliefs in a simpler way. We construct stationary strategies which have the property that each player is indifferent among each of his actions no matter what private history his opponnent has observed. It is then irrelevant

\footnotetext{
${ }^{3}$ Bhaskar (1999) sharpens the analysis and shows that the payoff restriction used in Sekiguchi (1997) is not necessary. When public correlation devices are available, these strategies can also be used to prove the folk theorem.

${ }^{4}$ There is still an additional complication to overcome in establishing that a strict equilibrium is robust to approximate public monitoring. Conditional beliefs must move continuously uniformly across the infinite set of histories.
} 
how conditional beliefs are altered by the monitoring imperfections since the players' continuation strategies will be a best-reply to every conditional belief. This in turn implies that our strategies will be robust to all monitoring imperfections, including conditional independence and even negative correlation. We show that a large set of payoffs, including mutual cooperation, can be supported by such equilibria. However, not all individually rational payoffs can be supported in this way. To obtain the full folk theorem set of payoffs, we show how these robust equilibria generate continuation values that can enforce behavior yielding payoffs outside the original set for a sufficiently long but finite length of time.

A recent paper by Piccione (1998) employs a technique similar to ours. In the repeated prisoners' dilemma Piccione uses dynamic programming techniques over the infinite state-space of private histories to construct the mixed strategies necessary to maintain indifference. His strategies can be used to approximate most of the feasible, individually rational payoff set. Our approach uses stationary behavior strategies that condition only on one period of history. This dramatically simplifies the dynamic program yielding a system of four equations which can easily be solved for equilibrium mixtures. ${ }^{5}$ Beyond the computational simplicity, the simple structure makes this a more promising direction for results in more general games. We demonstrate the flexibility of our approach by analyzing some more general games in section 4 .

In section 1, the perfect monitoring folk theorem is proven for a large subset of the feasible payoff set using simple two state mixed strategies. In section 2, it is shown that the strategies used are robust, and the limit folk theorem is established for these payoffs. In section 3 we show how to augment these strategies to obtain the full set of feasible individually rational payoffs. In section 4, we consider more general games. For 2 player games, we provide a sufficient condition on stage payoffs under which our approach will apply. We also analyze a symmetric $N$-player prisoners' dilemma and show how to extend our approach and obtain nearly cooperative payoffs for high discount factors and sufficiently accurate monitoring. Finally, section 5 concludes.

\footnotetext{
${ }^{5}$ We also obtain the full set of payoffs exactly.
} 


\section{$1 \quad$ Perfect Monitoring}

We consider the $\delta$-discounted infinitely repeated prisoner's dilemma, the normalized stage-game payoffs of which are displayed below, where $g, l>0$.

\begin{tabular}{l|c|c|}
\multicolumn{1}{c}{} & \multicolumn{1}{c}{$C$} & \multicolumn{1}{c}{$D$} \\
\cline { 2 - 3 }$C$ & 1,1 & $-l, 1+g$ \\
\cline { 2 - 3 }$D$ & $1+g,-l$ & 0,0 \\
\cline { 2 - 3 } & &
\end{tabular}

Figure 1: Normalized Prisoners' Dilemma

In this section, monitoring is assumed perfect. ${ }^{6}$ Consider the family of behavior strategies defined as follows. Player $i$ 's $(i=1,2)$ plays $C$ in stage 1 , and in any subsequent stage $t$, his mixed action depends only on the outcome in stage $t-1$. Denote by $\pi_{a_{i} a_{j}}^{i}$ the probability with which player $i$ plays $C$ conditional on the outcome $\left(a_{i}, a_{j}\right)$ occurring in the previous period. Note that the behavior in period $t>1$ is independent of $t$. For consistency with the following section, we will use lower-case subscripts, e.g. $\pi_{c d}^{j}$. Below is a diagram of the two-state machine that plays this strategy. ${ }^{7}$

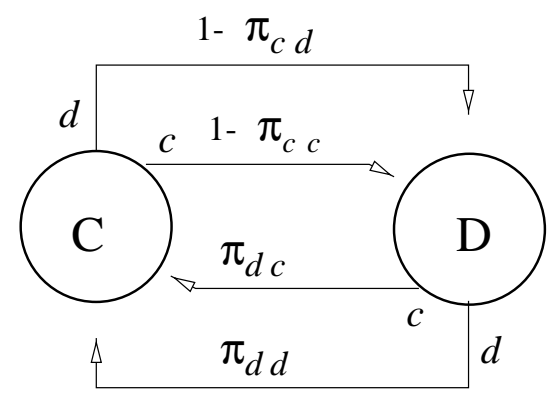

Figure 2: Machine representation of $\pi^{i}$. To avoid clutter, we have left out the arrows that return to the preceding state.

\footnotetext{
${ }^{6}$ Another interesting question concerns the robustness of the folk theorem under imperfect public monitoring: what payoffs in the public monitoring game can be obtained when monitoring becomes nearly public, but remains imperfect? The techniques here can be extended to this context, and is a subject for further research.

${ }^{7}$ Obara (1999a) independently applied strategies of this form to repeated games with public monitoring.
} 
We will show that for any payoff pair $\left(v_{1}, v_{2}\right)$ in the square $V=(0,1] \times$ $(0,1]$, there are strategies of this form that constitute an equilibrium of the repeated game (assuming sufficient patience) and obtain average payoffs $\left(v_{1}, v_{2}\right)$. In the next section, we will show that equilibria of this form are robust to private monitoring. Obviously $V$ is not the entire feasible, individually-rational set. The construction will make it clear that more cannot be achieved using strategies of this simple form. In section 3 we show how to modify these strategies to obtain any feasible individually rational payoff in a robust equilibrium. Let $V^{\circ}$ denote the interior of $V$.

Fix values $V_{C}^{i}$ and $V_{D}^{i}$ in $(0,1]$, with $V_{C}^{i}>V_{D}^{i}$ for $i=1,2$, and let $\bar{\delta}<1$ satisfy $\min _{i}\left\{V_{C}^{i}-V_{D}^{i}\right\}>1-\bar{\delta}$. The goal is to find $\delta \in(\bar{\delta}, 1)$ sufficiently large and to construct probabilities $\pi^{j}$ such that (1) in any period in which $j$ is playing $C$, player $i$ is indifferent between actions $C$ and $D$ and obtains continuation value $V_{C}^{i}$ and (2), in any period in which player $j$ is playing $D$, player $i$ is indifferent between actions $C$ and $D$ and obtains continuation value $V_{D}^{i}$. From this it will follow that each player is indifferent between $C$ and $D$ after every history (a fact that is essential for the robustness argument), and player $i$ gets average payoff $V_{C}^{i}$, for $i=1,2$. The following four equations express these conditions:

$$
\begin{aligned}
V_{C}^{i} & =(1-\delta)+\delta\left[\pi_{c c}^{j} V_{C}^{i}+\left(1-\pi_{c c}^{j}\right) V_{D}^{i}\right] \\
& =(1-\delta)(1+g)+\delta\left[\pi_{c d}^{j} V_{C}^{i}+\left(1-\pi_{c d}^{j}\right) V_{D}^{i}\right] \\
V_{D}^{i} & =-l(1-\delta)+\delta\left[\pi_{d c}^{j} V_{C}^{i}+\left(1-\pi_{d c}^{j}\right) V_{D}^{i}\right] \\
& =\delta\left[\pi_{d d}^{j} V_{C}^{i}+\left(1-\pi_{d d}^{j}\right) V_{D}^{i}\right]
\end{aligned}
$$

Equation 1 is equivalent to the following

$$
\pi_{c c}^{j}=\frac{V_{C}^{i}-(1-\delta)-\delta V_{D}^{i}}{\delta\left(V_{C}^{i}-V_{D}^{i}\right)}
$$

Because $V_{C}^{i}-\delta V_{D}^{i}>V_{C}^{i}-V_{D}^{i}>1-\bar{\delta}>1-\delta$, the numerator and hence the fraction is greater than zero. It is no greater than one iff $V_{C}^{i}-(1-\delta) \leq \delta V_{C}^{i}$ which is equivalent to $V_{C}^{i} \leq 1$ which was assumed. For future reference, note that $\pi_{c c}^{j}$ can be made interior if $V_{C}^{i}<1$.

Combining 1 and 2 and manipulating, we obtain

$$
\pi_{c c}^{j}-\pi_{c d}^{j}=\left(\frac{1-\delta}{\delta}\right)\left(\frac{g}{V_{C}^{i}-V_{D}^{i}}\right)
$$


Since $V_{C}^{i}-V_{D}^{i}>0$, this is greater than zero and can be made arbitrarily small by choosing $\delta$ sufficiently close to 1 . Thus, $\pi_{c d}^{j} \in(0,1)$ for sufficiently large $\delta$. Note also that for any such $\delta$, this $\pi_{c d}^{j} \in(0,1)$ continues to hold for any $\hat{V}_{C}^{i}>V_{C}^{i}$.

We have shown that then there exist probabilities $\pi_{c c}^{j} \in(0,1]$ and $\pi_{c d}^{j} \in$ $(0,1)$ such that if $V_{D}^{i}$ is the value to player $i$ when his opponent is playing $D$, then $V_{C}^{i}$ is the value to $i$ when his opponent is playing $C$, and $i$ is indifferent between $C$ and $D$ after such histories.

Now equation 4 reduces to

$$
\pi_{d d}^{j}=\left(\frac{1-\delta}{\delta}\right)\left(\frac{V_{D}^{i}}{V_{C}^{i}-V_{D}^{i}}\right)
$$

This is always positive and will be less than 1 once $\delta$ is sufficiently close to 1. Finally, equations 3 and 4 combine to yield

$$
\pi_{d c}^{j}-\pi_{d d}^{j}=\left(\frac{1-\delta}{\delta}\right)\left(\frac{l}{V_{C}^{i}-V_{D}^{i}}\right)
$$

which again makes $\pi_{d c}^{j}$ an interior probability once $\delta$ is sufficiently large. Again, if we fix such $\delta, \pi_{d c}^{j}$ will remain interior for any $V_{C}^{i}$ closer to 1 . Thus, interior probabilities can be found to ensure that when player $j$ is playing $D$, player $i$ is indifferent between $C$ and $D$ and obtains a continuation value $V_{D}^{i}$.

We conclude that for $\delta$ sufficiently close to 1 , there exists a strategy for player $j$ satisfying equations $1-4$ for any value $\hat{V}_{C}^{i} \in\left[V_{C}^{i}, 1\right]$. By the symmetric argument, there exists a strategy for player $i$ which satisfy the analogous equations for player $j$. Furthermore, since these equations imply that each player is indifferent between $C$ and $D$ after every history, each strategy is a best-response to the other after every history. Thus, the strategies form a subgame-perfect equilibrium of the perfect monitoring game and since each strategy is assumed to play $C$ in the first stage, player $i$ obtains average payoff $v_{i}=V_{C}^{i}$.

\section{A Limit Folk Theorem}

The focus now turns to the repeated prisoners' dilemma with private monitoring. In this setting, players do not directly observe the actions chosen by their opponent. Following each stage of play, each player observes his own 
chosen action and a private signal which depends on the outcome in that stage. Let $\Sigma_{i}$ be a finite set of signals for player $i$, and $\Sigma=\times_{i} \Sigma_{i}$. Assume that each $\Sigma_{i}$ has at least two elements.

A monitoring technology is a collection $\left\{m(\cdot \mid a): a \in\{C, D\}^{2}\right\}$ of probability distributions over signal profiles, one for each possible stage-game outcome. The marginal distribution over player $i$ 's signal will be denoted $m^{i}(\cdot \mid a)$. Perfect monitoring corresponds to a technology $m_{0}$ which satisfies two conditions. First, each $m_{0}(\cdot \mid a)$ exhibits perfect correlation (public monitoring); and second, for each $i$ there is a set $c^{i} \subset \Sigma_{i}$ such that if $a_{j}=C$ then $m_{0}^{i}\left(c^{i} \mid a\right)=1$ and if $a_{j}=D$ then $m_{0}^{i}\left(c^{i} \mid a\right)=0$.

Say that a monitoring technology is an $\varepsilon$-perturbation of $m^{0}$ if for each $i$ there is a set $c^{i} \subset \Sigma_{i}$ such that $a_{j}=C$ implies $m^{i}\left(c^{i} \mid a\right)>1-\varepsilon$ and $a_{j}=D$ implies $m^{i}\left(c^{i} \mid a\right)<\varepsilon$. Note that this definition involves no requirement on the correlation in $m$, and in particular, includes as a special case, independent private monitoring: technologies $m$ for which $m^{i}(\cdot \mid a)$ is independent of $m^{j}(\cdot \mid a)$ for each $a$. Henceforth, we restrict attention to technologies that are perturbations of $m_{0}$ and write $m_{a_{i} a_{j}}^{i}=m^{i}\left(c^{i} \mid a_{i}, a_{j}\right)$, so for example $m_{D C}^{i}$ represents the probability that $i$ observes a signal in $c^{i}$ when $i$ plays $D$ and $j$ plays $C$. Let $d^{i}$ denote the complement in $\Sigma_{i}$ of $c^{i}$.

The $\delta$-discounted repeated prisoners' dilemma with private monitoring technology $m$ will be denoted $G^{\infty}(\delta, m)$. A strategy $\pi^{i}$ in $G^{\infty}(\delta, m)$ specifies a mixed action for each history of own-actions and observed signals. We will restrict attention to strategies which depend only on histories of length 1 and condition only on the player's own action and the events $c^{i}$ and $d^{i}$. By analogy to the previous section, write e.g. $\pi_{d c}^{i}$ for the probability with which player $i$ plays $C$ following a stage in which $i$ played $D$ and observed a signal in $c^{i}$. Set $\pi_{\emptyset}^{i}=1$.

For any two strategy profiles $\pi, \tilde{\pi}$ of this form, define the distance $|\pi-\tilde{\pi}|$ to be $\max \left|\pi_{s}^{i}-\tilde{\pi}_{s}^{i}\right|$ where the maximum is over players $i=1,2$ and states $s$ of the machine.

Definition 1 A subgame-perfect equilibrium $\pi$ of $G^{\infty}\left(\delta, m_{0}\right)$ is robust to private monitoring if for every $e>0$ there exists $\bar{\varepsilon}>0$ such that for all $\varepsilon \in(0, \bar{\varepsilon})$ and all $\varepsilon$-perturbations $m_{\varepsilon}$ of $m_{0}$, there is a sequential equilibrium $\tilde{\pi}$ of $G^{\infty}\left(\delta, m_{\varepsilon}\right)$, within e distance of $\pi$ and with payoffs within e of the payoffs under $\pi$.

A sufficient condition for a pair $\left(\pi^{1}, \pi^{2}\right)$ to be a sequential equilibrium 
of $G^{\infty}(\delta, m)$ with payoffs $\left(v_{1}, v_{2}\right)$ is for the mixtures to solve the following equations for $V_{C}^{i}=v_{i}, i=1,2, j \neq i$.

$$
\begin{gathered}
V_{C}^{i}=(1-\delta)+\delta V_{C}^{i}\left[m_{C C}^{j} \pi_{c c}^{j}+\left(1-m_{C C}^{j}\right) \pi_{c d}^{j}\right] \\
+\delta V_{D}^{i}\left[\left(1-m_{C C}^{j}\right)\left(1-\pi_{c d}^{j}\right)+m_{C C}^{j}\left(1-\pi_{c c}^{j}\right)\right] \\
\begin{aligned}
& V_{C}^{i}=(1-\delta)(1+g)+\delta V_{C}^{i}\left[m_{C D}^{j} \pi_{c c}^{j}+\left(1-m_{C D}^{j}\right) \pi_{c d}^{j}\right] \\
&+\delta V_{D}^{i}\left[\left(1-m_{C D}^{j}\right)\left(1-\pi_{c d}^{j}\right)+m_{C D}^{j}\left(1-\pi_{c c}^{j}\right)\right] \\
& V_{D}^{i}=-l(1-\delta)+\delta V_{C}^{i}\left[m_{D C}^{j} \pi_{d c}^{j}+\left(1-m_{D C}^{j}\right) \pi_{d d}^{j}\right] \\
& \quad+\delta V_{D}^{i}\left[\left(1-m_{D C}^{j}\right)\left(1-\pi_{d d}^{j}\right)+m_{D C}^{j}\left(1-\pi_{d c}^{j}\right)\right] \\
& V_{D}^{i}=\delta V_{C}^{i}\left[m_{D D}^{j} \pi_{d c}^{j}+\left(1-m_{D D}^{j}\right) \pi_{d d}^{j}\right] \\
& \quad+\delta V_{D}^{i}\left[\left(1-m_{D D}^{j}\right)\left(1-\pi_{d d}^{j}\right)+m_{D D}^{j}\left(1-\pi_{d c}^{j}\right)\right]
\end{aligned}
\end{gathered}
$$

Equations 5 and 6 state that player $i$ gets average payoff $V_{C}^{i}$ when he plays either $C$ or $D$ in any stage in which player $j$ plays $C$. Equations 5 and 6 state that player $i$ gets average payoff $V_{D}^{i}$ when he plays either $C$ or $D$ in any stage in which player $j$ plays $D$. If these equations are satisfied, then player $i$ is always indifferent between his two actions and is therefore willing to play any mixed strategy after every history.

When monitoring is perfect, $m_{*, D}^{j}=1-m_{*, C}^{j}=0$ and these equations reduce to equations 1-4 from the previous section. There it was shown that provided $\delta$ is sufficiently large and $V_{C}^{i} \in(0,1]$, a solution $\left(\bar{\pi}^{j}, \bar{V}_{C}^{i}, \bar{V}_{D}^{i}\right)$ exists , and that the mixtures $\bar{\pi}_{c d}^{j}$ and $\bar{\pi}_{d c}^{j}$ can be chosen to be interior. We can now fix $\bar{\pi}_{c c}^{j}$ and $\bar{\pi}_{d d}^{j}$ at the solution and solve for $V_{C}^{i}, V_{D}^{i}, \pi_{c d}^{j}$, and $\pi_{d c}^{j}$ :

$$
\begin{aligned}
\pi_{d c}^{j} & =\frac{\delta \pi_{d d}^{j} \mu_{D}\left(\mu_{C}-g\left(1-m_{C C}^{j}\right)\right)+l\left(1-\delta\left(\bar{\pi}_{c c}^{j}-\bar{\pi}_{d d}^{j}\right)+\pi_{d d}^{j} m_{D D}^{j}\right)}{\delta g \mu_{D}\left(m_{C C}^{j}-1\right)+\mu_{C}\left(\mu_{D}-l m_{D D}^{j}\right)} \\
\pi_{c d}^{j} & =\frac{\delta \mu_{C} \pi_{c c}^{j}\left(\mu_{D}+l \pi_{d d}^{j}\right)+g \mu_{D}\left(\delta\left(\pi_{c c}^{j} m_{C C}^{j}-\pi_{d d}^{j}\right)-1\right)}{\delta g \mu_{D}\left(m_{C C}^{j}-1\right)+\mu_{C}\left(\mu_{D}-l m_{D D}^{j}\right)} \\
V_{C}^{i} & =\bar{V}_{C}^{i}+\frac{g \mu_{D}\left(m_{C C}^{j}-1\right)\left(1+\delta\left(\pi_{d d}^{j}-1\right)\right)-\delta \mu_{C} l\left(\pi_{c c}^{j}-1\right) m_{D D}^{j}}{\mu_{C} \mu_{D}\left(\delta\left(\bar{\pi}_{c c}^{j}-\bar{\pi}_{d d}^{j}\right)-1\right)} \\
V_{D}^{i} & =\bar{V}_{D}^{i}+\frac{\delta\left[g \mu_{D} \pi_{d d}^{j}\left(1-m_{C C}^{j}\right)-l \mu_{C} \pi_{c c}^{j} m_{D D}^{j}\right]-l \mu_{C} m_{D D}^{j}}{\mu_{C} \mu_{D}\left(\delta\left(\bar{\pi}_{c c}^{j}-\bar{\pi}_{d d}^{j}\right)-1\right)}
\end{aligned}
$$

where we have written $\mu_{D}=m_{D C}^{j}-m_{D D}^{j}$ and $\mu_{C}=m_{C C}^{j}-m_{C D}^{j}$. These equations define the left-hand side variables as continuous functions of the 
monitoring parameters. Since $\bar{\pi}_{c d}^{j}$ and $\bar{\pi}_{d c}^{j}$ were interior, it follows that for $m_{*, D}^{j}$ and $1-m_{*, C}^{j}$ sufficiently close to zero, there exists a solution $\left(\pi^{j}, V_{D}^{i}, V_{C}^{i}\right)$ to equations 5-8 with mixtures $\pi^{j}$ and values $V_{C}^{i}, V_{D}^{i}$ arbitrarily close to $\left(\bar{\pi}^{j}, \bar{V}_{C}^{i}, \bar{V}_{D}^{i}\right)$. Player $j$ therefore has a strategy against which every strategy of player $i$ is a best-response and achieves payoff $V_{C}^{i}$. This establishes the following theorem.

Theorem 1 (Robust folk theorem) Let $\left(v_{1}, v_{2}\right) \in V$. There exist $\bar{\delta} \in$ $(0,1)$ such that for all $\delta \in(\bar{\delta}, 1)$, there exists a robust subgame perfect equilibrium of $G^{\infty}\left(\delta, m_{0}\right)$ with payoffs $\left(v_{1}, v_{2}\right)$.

Now suppose $V_{C}^{i} \in(0,1)$ and recall that this implies that $\bar{\pi}_{c c}^{j} \in(0,1)$. We can now fix $\left(\bar{V}_{C}^{i}, \bar{V}_{D}^{i}\right) \in(0,1)^{2}$ and solve equations 5-8 for the following explicit relations between the equilibrium strategies and the monitoring probabilities.

$$
\begin{aligned}
\pi_{c c}^{j} & =\frac{\left(V_{C}^{i}-\delta V_{D}^{i}\right)\left(m_{C C}^{j}-m_{C D}^{j}\right)+(1-\delta)\left(g+m_{C D}^{j}-m_{C C}^{j}(1+g)\right)}{\delta\left(m_{C C}^{j}-m_{C D}^{j}\right)\left(V_{C}^{i}-V_{D}^{i}\right)} \\
\pi_{c d}^{j} & =\frac{\left(V_{C}^{i}-\delta V_{D}^{i}\right)\left(m_{C C}^{j}-m_{D D}^{j}\right)+(1-\delta)\left(m_{C D}^{j}-(1+g) m_{C C}^{j}\right)}{\delta\left(m_{C C}^{j}-m_{C D}^{j}\right)\left(V_{C}^{i}-V_{D}^{i}\right)} \\
\pi_{d c}^{j} & =\frac{(1-\delta)\left[l\left(1-m_{D D}^{j}\right)-V_{D}^{i}\left(m_{D D}^{j}-m_{D C}^{j}\right)\right]}{\delta\left(m_{D C}^{j}-m_{D D}^{j}\right)\left(V_{C}^{i}-V_{D}^{i}\right)} \\
\pi_{d d}^{j} & =\frac{(1-\delta)\left[\left(m_{D C}^{j}-m_{D D}^{j}\right) V_{D}^{i}-l m_{D D}^{j}\right]}{\delta\left(m_{D C}^{j}-m_{D D}^{j}\right)\left(V_{C}^{i}-V_{D}^{i}\right)}
\end{aligned}
$$

One can easily verify that these define equilibrium behavior strategies as continuous functions of the monitoring probabilities $m^{j}$. Since all mixtures in $\pi^{j}$ are interior, it follows that for $m_{*, D}^{j}$ and $1-m_{*, C}^{j}$ sufficiently close to zero, equations 5-8 can be solved for probabilities $\pi^{j}$, and the symmetric set of equations can be solved for probabilities $\pi^{i}$. These solutions will be Nash equilibria of the private monitoring game with values $\left(\bar{V}_{C}^{1}, \bar{V}_{C}^{2}\right)$. This proves the limit folk theorem

Theorem 2 (Limit folk theorem) Let $\left(v_{1}, v_{2}\right) \in V^{\circ}$. There exist $\bar{\delta}, \bar{\varepsilon} \in$ $(0,1)$ such that for all $\delta \in(\bar{\delta}, 1)$ and $\varepsilon \in(0, \bar{\varepsilon})$, if $m$ is an $\varepsilon$-perturbation of $m_{0}$, then there exists a sequential equilibrium of $G^{\infty}(\delta, m)$ with payoffs $\left(v_{1}, v_{2}\right)$. 
The order of quantification was not proven above. In the previous section, it was shown that if there is a solution for some $\delta$ then there is a solution for any larger $\hat{\delta}$. One can easily verify that the same holds here: if there are probabilities that solve equations 5-8 for some $\varepsilon$ and $\delta$, then there are probabilities that solve the equations for $\varepsilon$ and any $\hat{\delta}>\delta$.

\section{Extending to the Full Payoff Set}

We have shown that any payoff pair in $V$ can be obtained in a robust equilibrium. This leaves out much of the feasible, individually rational set of payoffs. In particular, the only efficient payoff pair in $V$ is the symmetric payoff $(1,1)$. Unfortunately, nothing outside of $V$ can be obtained robustly using strategies of the simple form considered above. To see this, note that if in equilibrium, player $i$ is indifferent between $C$ and $D$ after every history, then his long-run average payoff must be equal to the payoff he would get by playing $C$ after every history. Obviously such a payoff cannot exceed 1 .

However, more can be achieved using more complicated (yet still finitestate) strategies. We sketch the idea here, and prove it formally below. Return to the perfect monitoring case. Fix $V_{D}^{1}>0$ close to zero and $V_{C}^{2}=1$ Let $V_{C}^{1}$ be any value in $\left(V_{D}^{1}, 1\right]$. We have shown in theorem 1 that for $\delta$ close enough to 1 , there exist robust equilibrium strategies $\left(\pi^{1}, \pi^{2}\right)$ which obtain values $\left(V_{C}^{1}, V_{C}^{2}\right)$, and player 2's strategy can be chosen so that player 1 obtains value $V_{D}^{1}$ whenever player 2 plays $D$.

Consider the following strategies. Player 1 plays $C$ in stage 1 , then proceeds with $\pi^{1}$ starting in stage 2 . Player 2 plays $D$ in stage 1 . In stage 2 , player 2 switches to strategy $\pi^{2}$ but starts in state $D$ if he observes that player 1 has played $D$ in stage 1 . If 2 observes that 1 has played $C, 2$ starts in state $C$.

Let $\left(v_{1}, v_{2}\right)=(1-\delta)(-l, 1+g)+\delta\left(V_{C}^{1}, V_{C}^{2}\right)$. The above strategies form an equilibrium with payoffs $\left(v_{1}, v_{2}\right)$ if the following incentive constraints are satisfied.

$$
\begin{aligned}
(1-\delta)(1+g)+\delta V_{C}^{1} & >(1-\delta)+\delta V_{C}^{1} \\
(1-\delta)(-l)+\delta V_{C}^{2} & >\delta V_{D}^{2}
\end{aligned}
$$

These hold for sufficiently large $\delta$. Note that the payoffs $\left(v_{1}, v_{2}\right)$ are outside of $V$. Now we claim that this equilibrium is robust to private monitoring. To see this, note that in stage two, each player is indifferent between his 
two actions regardless of the outcome in stage 1. Each is therefore willing to behave as the strategy demands even after erroneous signals. The continuation payoffs $V_{C}^{1}, V_{C}^{2}, V_{D}^{1}$ are continuous in the monitoring perturbation because the continuation strategies are robust. Thus, because the first-stage incentive constraints are satisfied with strict inequalities, they will continue to hold for small perturbations of $m_{0}$. We show below that by increasing the number of stages in which $(C, D)$ is played, any feasible individually rational payoff vector can be sustained in a robust equilibrium.

To prove this formally, we make an additional assumption. We assume that $g-l \leq 1$ so that mutual cooperation is not Pareto-dominated by any other profile. We make this assumption merely to simplify the exposition as this allows us to achieve any individually rational payoff by a convex combination of the set $V$ and a single additional point. Our result continues to hold without this assumption, with strategies alternating between $(C, D)$ and $(D, C)$, slightly complicating the proof.

For $P \subset \mathbf{R}^{n}$, denote by $\operatorname{Co}(P)$, the convex hull of $P$.

Lemma 1 Let $P$ be a convex subset of $R^{n}$ with a non-empty interior amd $v \in R^{n}$. For any $u \in$ int $\operatorname{co}(P \cup\{v\})$, there is a $\bar{\delta}$ such that for all $\delta \in(\bar{\delta}, 1]$, there is a natural number $N$, and $w \in P$ such that

$$
u=\left(1-\delta^{N}\right) v+\delta^{N} w
$$

Proof: Fix $u \in$ int $c o(P \cup\{v\})$. Since $P$ is convex with a non-empty interior, there is a $\lambda^{\prime} \in(0,1)$ and a $w^{\prime} \in$ int $P$ such that

$$
u=\left(1-\lambda^{\prime}\right) v+\lambda^{\prime} w^{\prime}
$$

Since $w^{\prime} \in$ int $P$, there is (by continuity) an $\varepsilon>0$ (and $\varepsilon<\lambda^{\prime}$ ), such that for all $\lambda \in B_{\varepsilon}\left(\lambda^{\prime}\right)$, there is a $w(\lambda) \in P$ satisfying

$$
u=(1-\lambda) v+\lambda w(\lambda)
$$

Let $N(\delta)=\max \left\{n \mid \delta^{n} \geq \lambda^{\prime}\right\}$. The remaining task is hence to show that there is a $\bar{\delta}$ such that for all $\delta \in(\bar{\delta}, 1], \delta^{N(\delta)} \in B_{\varepsilon}\left(\lambda^{\prime}\right)$. By construction, $\delta^{N(\delta)+1}<\lambda^{\prime}$ and hence $\delta^{N(\delta)}<\frac{\lambda^{\prime}}{\delta}$ or $\delta^{N(\delta)}-\lambda^{\prime}<\frac{\lambda^{\prime}}{\delta}-\lambda^{\prime}=\lambda^{\prime}\left(\frac{1}{\delta}-1\right)$ and the claim holds for $\bar{\delta}=\frac{\lambda^{\prime}-\varepsilon}{\lambda^{\prime}}$.

Theorem 3 For each $v \in V^{*}$ there is a $\bar{\delta} \in(0,1)$ such that for all $\delta \in(\bar{\delta}, 1)$ there is a robust subgame perfect equilibrium of $G^{\infty}\left(\delta, m_{0}\right)$ with payoffs $v$. 


\section{Proof:}

Let $V_{1}^{*}$ be the subset of $V^{*}$ in which player 1's payoff is at least 1 (and hence player 2's payoff is less than 1). We will show that each $v \in V_{1}^{*}$ is a robust equilibrium payoff for $\delta$ close enough to 1 . Together with the symmetric argument when the players roles are reversed, this will establish the theorem.

If $v \in V_{1}^{*}$ then $v \in \operatorname{Co}(V \cup\{(1+g,-l)\}$ ) (see figure 3). By lemma 1 there is a $\bar{\delta}$ such that for all $\delta \in(\bar{\delta}, 1)$ there is a $N$ and $u \in V$ such that $v=\left(1-\delta^{N}\right)(1+g,-l)+\delta^{N} u$. Note that since $u_{2}>0>-l, u_{2}>v_{2}$. Choose $w_{2}$ so that $0<w_{2}<v_{2}$ and $\delta$ sufficiently close to 1 so that $u$ and $w:=\left(u_{1}, w_{2}\right)$ are robust equilibrium payoffs.

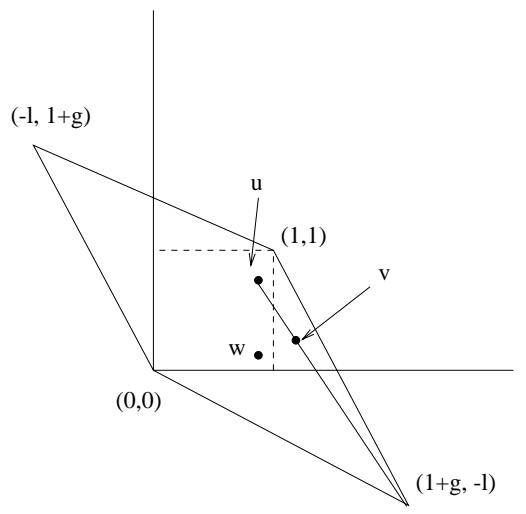

Figure 3: Prisoner's Dilemma feasiblepayoff set.

Consider the following strategy profile. Player 1 plays $D$ indpendent of history for the first $N$ periods. If player 1 observes that 2 has played $C$ in each of the first $N$ stages, player 1 continues with a strategy $s_{1}$ against which every strategy of player 2 is a best-response and achieves a payoff of $u_{2}$. Otherwise, player 1 continues in stage $N+1$ with a strategy $s_{1}^{\prime}$ against which every strategy of player 2 achieves a payoff of $w_{2} .{ }^{8}$

Player 2 plays $C$ in each of the first $N$ stages provided he has never played $D$. If in any of the first $N-1$ stages, 2 has played $D$, then 2 continues with $D$ through stage $N$. Independent of history, beginning in stage $N+1,2$

\footnotetext{
${ }^{8}$ In a working paper version, we showed by a more complicated argument that less draconian strategies can be used.
} 
plays a strategy $s_{2}$ against which every strategy of player 1 achieves a payoff of $u_{1}$.

By construction, any continutation for player 1 is a best response to $s_{2}$ beginning in stage $N+1$. And since 2 continues with $s_{2}$ is independent of history, it is a best-response for 1 to play $D$ in each of the first $N$ stages.

Likewise, $s_{2}$ is a best response to both $s_{1}$ and $s_{1}^{\prime}$ beginning in stage $N+1$. To establish that this profile is a subgame perfect equilibrium, therefore, it remains only to check that 2 is willing to play according to the equilibrium in each of the first $N$ stages. If 2 has played $D$ prior to stage $N$, then independent of any future history, 1 will play $s_{1}^{\prime}$ and 2 will receive continuation payoff $w_{2}$. Therefore, 2 optimally continues with $D$ through stage $N$. Finally, suppose that in each stage up to $s \leq N, 2$ has played $C$. If 2 plays according to the equilibrium, his payoff is $-l\left(1-\delta^{N-s+1}\right)+\delta^{N-s+1} u_{2}>$ $-l\left(1-\delta^{N}\right)+\delta^{N} u_{2}=v_{2}$. If he instead plays $D$ in stage $s$ and then continues with his equilibrium strategy, his payoff is $\delta^{N-s+1} w_{2}<w_{2}$. Since $w_{2}<v_{2}$, player 2 optimally plays $C$.

To conclude the proof, we show that the above equilibrium is robust. By Theorem 1 , for any sufficiently small $\varepsilon$-perturbation of $m_{0}$, there are continuation strategies $\tilde{s}_{1}, \tilde{s}_{1}^{\prime}$, and $\tilde{s}_{2}$ arbitrarily close to $s_{1}, s_{1}^{\prime}$, and $s_{2}$, generating continuation payoffs arbitrarily close to $w_{1}, u_{1}$, and $u_{2}$ respectively, and making the opponent indifferent among all strategies. It follows that beginning in stage $N+1$, both $\tilde{s}_{1}$ and and $\tilde{s}_{1}^{\prime}$ are best-responses to $\tilde{s}_{2}$ and $\tilde{s}_{2}$ is a best-response for 2 whatever his belief over $s_{1}$ and $s_{1}^{\prime}$. The players will play as before for the first $N$ stages, and now continue with these continuation strategies. The payoffs in the first $N$ stages are unaffected by $\varepsilon$. The distribution over $N$-stage histories, and hence continuation payoffs beginning in stage $N+1$ is continuous in $\varepsilon$. Therefore $\varepsilon$ can be taken sufficiently small so that the overall payoffs to these strategies are arbitrarily close to $v$ and they remain a sequential equilibrium.

\section{Extensions}

In this section, we outline briefly how the method for finding robust subgame perfect equilibria can be extended to more general games. 


\subsection{Two-Player Games}

Let $G=\left(A_{1}, A_{2}, g_{1}, g_{2}\right)$ be a finite two- player normal form game, where $A_{i}$ are the action sets and $g_{i}$ payoff functions. Let $G^{\infty}(\delta)$ the infinitely repeated game with $G$ as the stage game and $\delta$ as the discount factor. Consider two strategy profiles $\left(a_{1}^{\prime}, a_{2}^{\prime}\right)$ and $\left(a_{1}^{\prime \prime}, a_{2}^{\prime \prime}\right)$ and assume that the following condition on the payoff functions is satisfied: For $i \in\{1,2\}$, there are $\underline{v}_{i}$ and $\bar{v}_{i}>\underline{v}_{i}$ such that

$$
\begin{gathered}
\text { i) } \max _{a_{i} \in A_{i}} g_{i}\left(a_{i}, a_{-i}^{\prime \prime}\right)<\underline{v}_{i} \text { and } \\
\text { ii) } \min \left\{g_{i}\left(a_{i}^{\prime}, a_{-i}^{\prime}\right), g_{i}\left(a_{i}^{\prime \prime}, a_{-i}^{\prime}\right)\right\}>\bar{v}_{i} \text {. }
\end{gathered}
$$

The first step is again the construction of a subgame perfect equilibrium in the game with perfect monitoring where the players mix between $\left(a_{i}^{\prime}, a_{i}^{\prime \prime}\right)$. In analogy to the previous sections, let $\pi_{a_{i}, a_{j}}^{i} \in \Delta\left(A_{i}\right)$ be the probability distribution on player $i^{\prime} s$ pure actions conditional on observing outcome $\left(a_{i}, a_{j}\right)$ at the previous stage, and let $V_{a_{i}}^{i}$ be as before.

Theorem 4 For any $v \in\left(\underline{v}_{1}, \bar{v}_{1}\right) \times\left(\underline{v}_{2}, \bar{v}_{2}\right)$, there is a $\bar{\delta}<1$ such that $v$ can be supported as SPE payoff in $G^{\infty}(\bar{\delta})$ for all $\delta>\bar{\delta}$ using strategies where $\pi_{a_{i}, a_{j}}^{i}\left(a_{i}^{\prime}\right)>0, \pi_{a_{i}, a_{j}}^{i}\left(a_{i}^{\prime \prime}\right)>0$ and $\pi_{a_{i}, a_{j}}^{i}\left(a_{i}\right)=0$ for $a_{i} \notin\left\{a_{i}^{\prime}, a_{i}^{\prime \prime}\right\}$.

Proof: Pick $V_{a_{j}^{\prime}}^{i}$ and $V_{a_{j}^{\prime \prime}}^{i}$ such that $\underline{v}_{i}<V_{a_{j}^{\prime \prime}}^{i}<V_{a_{j}^{\prime}}^{i}<\bar{v}_{i}$. Suppose that $j$ randomizes between $a_{j}^{\prime \prime}$ and $a_{j}^{\prime}$ in each period so that $\pi_{a_{j}, a_{i}}^{j}\left(a_{j}\right)=0$ for $a_{j} \notin\left\{a_{j}^{\prime}, a_{j}^{\prime \prime}\right\}$. The claim is proved if there are probabilities $\pi_{a_{j}, a_{i}}^{j}\left(a_{j}^{\prime \prime}\right)$ such that the following conditions hold:

$$
\begin{aligned}
V_{a_{j}^{\prime \prime}}^{i} & =(1-\delta) g_{i}\left(a_{i}^{\prime \prime}, a_{j}^{\prime \prime}\right)+\delta\left[\pi_{a_{j}^{\prime \prime}, a_{i}^{\prime \prime}}^{j}\left(a_{j}^{\prime \prime}\right) V_{a_{j}^{\prime \prime}}^{i}+\left(1-\pi_{a_{j}^{\prime \prime}, a_{i}^{\prime \prime}}^{j}\left(a_{j}^{\prime \prime}\right)\right) V_{a_{j}^{\prime}}^{i}\right] \\
& =(1-\delta) g_{i}\left(a_{i}^{\prime}, a_{j}^{\prime \prime}\right)+\delta\left[\pi_{a_{j}^{\prime \prime}, a_{i}^{\prime}}^{j}\left(a_{j}^{\prime \prime}\right) V_{a_{j}^{\prime \prime}}^{i}+\left(1-\pi_{a_{j}^{\prime \prime}, a_{i}^{\prime}}^{j}\left(a_{j}^{\prime \prime}\right)\right) V_{a_{j}^{\prime}}^{i}\right] .
\end{aligned}
$$

For all $a_{i} \notin\left\{a_{i}^{\prime}, a_{i}^{\prime \prime}\right\}$

$$
\begin{aligned}
V_{a_{j}^{\prime \prime}}^{i} & \geq(1-\delta) g_{i}\left(a_{i}, a_{j}^{\prime \prime}\right)+\delta\left[\pi_{a_{j}^{\prime \prime}, a_{i}}^{j}\left(a_{j}^{\prime \prime}\right) V_{a_{j}^{\prime \prime}}^{i}+\left(1-\pi_{a_{j}^{\prime \prime}, a_{i}}^{j}\left(a_{j}^{\prime \prime}\right)\right) V_{a_{j}^{\prime}}^{i}\right] \\
V_{a_{j}^{\prime}}^{i} & =(1-\delta) g_{i}\left(a_{i}^{\prime \prime}, a_{j}^{\prime}\right)+\delta\left[\pi_{a_{j}^{\prime}, a_{i}^{\prime \prime}}^{j}\left(a_{j}^{\prime \prime}\right) V_{a_{j}^{\prime \prime}}^{i}+\left(1-\pi_{a_{j}^{\prime}, a_{i}^{\prime \prime}}^{j}\left(a_{j}^{\prime \prime}\right)\right) V_{a_{j}^{\prime}}^{i}\right] \\
& =(1-\delta) g_{i}\left(a_{i}^{\prime}, a_{j}^{\prime}\right)+\delta\left[\pi_{a_{j}^{\prime}, a_{i}^{\prime}}^{j}\left(a_{j}^{\prime \prime}\right) V_{a_{j}^{\prime \prime}}^{i}+\left(1-\pi_{a_{j}^{\prime}, a_{i}^{\prime}}^{j}\left(a_{j}^{\prime \prime}\right)\right) V_{a_{j}^{\prime}}^{i}\right]
\end{aligned}
$$


and also for all $a_{i} \notin\left\{a_{i}^{\prime}, a_{i}^{\prime \prime}\right\}$,

$$
V_{a_{j}^{\prime}}^{i} \geq(1-\delta) g_{i}\left(a_{i}, a_{j}^{\prime}\right)+\delta\left[\pi_{a_{j}^{\prime}, a_{i}}^{j}\left(a_{j}^{\prime \prime}\right) V_{a_{j}^{\prime \prime}}^{i}+\left(1-\pi_{a_{j}^{\prime}, a_{i}}^{j}\left(a_{j}^{\prime \prime}\right)\right) V_{a_{j}^{\prime}}^{i}\right]
$$

Our condition on payoffs ensures $g_{i}\left(a_{i}, a_{j}^{\prime \prime}\right)<V_{a_{j}^{\prime \prime}}^{i}$ for all $a_{i} \in A_{i}$, and $g_{i}\left(a_{i}^{\prime \prime}, a_{j}^{\prime}\right)>V_{a_{j}^{\prime}}^{i}$, and $g_{i}\left(a_{i}^{\prime}, a_{j}^{\prime}\right)>V_{a_{j}^{\prime}}^{i}$.

The first set of equalities and inequalities can, in fact, be solved as a set of equalities, and we get:

$$
\pi_{a_{j}^{\prime \prime}, a_{i}}^{j}\left(a_{j}^{\prime \prime}\right)=\frac{V_{a_{j}^{\prime \prime}}^{i}-(1-\delta) g_{i}\left(a_{i}, a_{j}^{\prime \prime}\right)-\delta V_{a_{j}^{\prime}}^{i}}{\delta\left(V_{a_{j}^{\prime \prime}}^{i}-V_{a_{j}^{\prime}}^{i}\right)} .
$$

Since $V_{a_{j}^{\prime}}^{i}>V_{a_{j}^{\prime \prime}}^{i}$ and $g_{i}\left(a_{i}, a_{j}^{\prime \prime}\right)<V_{a_{j}^{\prime \prime}}^{i}, 0<\pi_{a_{j}^{\prime \prime}, a_{i}}^{j}\left(a_{j}^{\prime \prime}\right)<1$ whenever $\delta$ is sufficiently close to 1 .

The second set of equalities yields:

$$
\begin{aligned}
\pi_{a_{j}^{\prime}, a_{i}^{\prime \prime}}^{j}\left(a_{j}^{\prime \prime}\right) & =\frac{(1-\delta)\left(V_{a_{j}^{\prime}}^{i}-g_{i}\left(a_{i}^{\prime \prime}, a_{j}^{\prime}\right)\right)}{\delta\left(V_{a_{j}^{\prime \prime}}^{i}-V_{a_{j}^{\prime}}^{i}\right)} \\
\pi_{a_{j}^{\prime}, a_{i}^{\prime}}^{j}\left(a_{j}^{\prime \prime}\right) & =\frac{(1-\delta)\left(V_{a_{j}^{\prime}}^{i}-g_{i}\left(a_{i}^{\prime}, a_{j}^{\prime}\right)\right)}{\delta\left(V_{a_{j}^{\prime \prime}}^{i}-V_{a_{j}^{\prime}}^{i}\right)} .
\end{aligned}
$$

Again, $\pi_{a_{j}^{\prime}, a_{i}^{\prime \prime}}^{j}\left(a_{j}^{\prime \prime}\right)$ and $\pi_{a_{j}^{\prime}, a_{i}^{\prime}}^{j}\left(a_{j}^{\prime \prime}\right)$ are probabilities for $\delta$ sufficiently close to 1 .

The second set cannot be satisfied as equalities, in general. To see this solve for the probabilities:

$$
\pi_{a_{j}^{\prime}, a_{i}}^{j}\left(a_{j}^{\prime \prime}\right)=\frac{(1-\delta)\left(V_{a_{j}^{\prime}}^{i}-g_{i}\left(a_{i}, a_{j}^{\prime}\right)\right)}{\delta\left(V_{a_{j}^{\prime \prime}}^{i}-V_{a_{j}^{\prime}}^{i}\right)} .
$$

If $g_{i}\left(a_{i}, a_{j}^{\prime}\right)<V_{a_{j}^{\prime}}^{i}$, and $V_{a_{j}^{\prime \prime}}^{i}<V_{a_{j}^{\prime}}^{i}$, the solution is negative.

This does not cause any problems, however, since we can pick an arbitrary $\bar{V}^{i}$ with $V_{a_{j}^{\prime \prime}}^{i}<\bar{V}^{i}<V_{a_{j}^{\prime}}^{i}$ and probabilities $\pi_{a_{j}^{\prime}, a_{i}}^{j}\left(a_{j}^{\prime \prime}\right)$ that yield for all $a_{i} \notin$ $\left\{a_{i}^{\prime}, a_{i}^{\prime \prime}\right\}$ :

$$
\bar{V}^{i}=(1-\delta) g_{i}\left(a_{i}, a_{j}^{\prime}\right)+\delta\left[\pi_{a_{j}^{\prime}, a_{i}}^{j}\left(a_{j}^{\prime \prime}\right) V_{a_{j}^{\prime \prime}}^{i}+\left(1-\pi_{a_{j}^{\prime}, a_{i}}^{j}\left(a_{j}^{\prime \prime}\right)\right) V_{a_{j}^{\prime}}^{i}\right] .
$$


A similar set of conditions holds for player $j$, and given that each player is indifferent after each history between actions $a_{i}^{\prime}$ and $a_{i}^{\prime \prime}$, and given that all other actions yield a weakly lower payoff, the randomizations above are best responses.

An argument similar to that in the previous sections can be given to show that there is, in fact, a robust subgame perfect equilibrium that yields $v$ as the payoff vector. Two corollaries follow immediately from the result above. Let $e=\left(e_{1}, e_{2}\right)$ be a pure strategy Nash equilibrium profile of the stage game. Denote the stage game equilibrium payoffs by $v_{1}^{e}$ and $v_{2}^{e}$ respectively.

Corollary 1 If there is a profile $a=\left(a_{1}, a_{2}\right)$ such that $\min \left\{g_{i}\left(e_{i}, a_{-i}\right), g_{i}\left(e_{i}, e_{-i}\right)\right\}>$ $v_{i}^{e}$ for $i \in\{1,2\}$, then the set of robust subgame perfect equilibrium payoffs has a non empty interior. Furthermore, we can require that player $i$ uses at each stage either $a_{i}$ or $e_{i}$ for $i=1,2$.

As an example of this corollary, consider the discretized Cournot competition model with linear demands. For concreteness, let $A_{i}=\{0,1,2, \ldots, 20\}$ and $g_{i}\left(a_{i}, a_{j}\right)=a_{i}\left(20-a_{i}-a_{j}\right)$. A Nash equilibrium of the stage game is $e_{i}=e_{j}=7$. The monopoly price is supported by action profile $a_{1}=a_{2}=5$. Observe that $g_{i}(e)=42, g_{i}(a)=50$ and $g_{i}\left(a_{i}, e_{j}\right)=56$. As a result, we conclude that any $v \in(42,50)^{2}$ can be supported as a robust subgame perfect equilibrium for sufficiently high $\delta$.

For the second corollary, let $m_{i} \in A_{i}$ be the action that minmaxes player $j$. Let $v_{i}$ denote the pure strategy minmax payoff of each player.

Corollary 2 If there is an $\left(a_{1}, a_{2}\right)$ such that $\min \left\{g_{i}\left(m_{i}, a_{-i}\right), g_{i}\left(a_{i}, a_{-i}\right)\right\}>$ $v_{i}$ for $i \in\{1,2\}$, then the set of robust subgame perfect equilibrium payoffs has a non empty interior. Furthermore, we can require that player $i$ uses at each stage either $a_{i}$ or $m_{i}$.

Using this corollary, it is easy to see that in some games, payoffs below those resulting from the unique dominant strategy equilibrium of the stage game are sustainable in robust subgame perfect equilibrium and hence in games with small imperfections in the monitoring technologies. Consider e.g. figure 4 .

In this example, $m_{i}=a_{2}$ for $i=1,2$. Hence the Corollary above implies that any $v \in(-1,0)^{2}$ can be supported as a robust equilibrium payoff. Observe also that the pure strategy Nash equilibrium payoff cannot be approximated by a mixed strategy equilibrium of the type as described above. ${ }^{9}$

\footnotetext{
${ }^{9}$ Although such payoffs can be obtained by strategies which play $\left(a_{1}, a_{1}\right)$ for sufficiently
} 


\begin{tabular}{ccc|}
\multicolumn{1}{c}{} & \multicolumn{1}{c}{$a_{1}$} & \multicolumn{1}{c}{$a_{2}$} \\
\cline { 2 - 3 }$a_{1}$ & 1,1 & $-1,0$ \\
\cline { 2 - 3 }$a_{2}$ & $0,-1$ & $-2,-2$ \\
\cline { 2 - 3 } & &
\end{tabular}

Figure 4: Payoffs below the dominant strategy equilibrium can be supported.

\subsection{N-Player Case}

We conclude this section by analyzing an example of a symmetric $N$-player game. The basic difficulty in comparison to the two player case is that the transitions between the states of the machines equivalent to those described in the previous section are no longer linear in the randomizations of the other players (even after conditioning on own actions). As a result, the probabilities cannot be solved for as before by simple linear algebra. In this subsection, we show that for $\delta$ close to unity, we can recover approximate linearity and show the robustness by an application of the implicit function theorem. ${ }^{10}$

We consider here the following version of the $N$-player Prisoner's Dilemma. $A_{i}=\{C, D\}$ for $i=1, \ldots, N$, and $g_{i}\left(C, a_{-i}\right)=n_{i}\left(a_{-i}\right)=g_{i}\left(D, a_{-i}\right)+1$, where $n_{i}\left(a_{-i}\right)$ is the number of players different from $i$ that play $C$ in profile $a_{-i}$. Let $G^{\infty}(\delta)$ stand for the infinitely repeated version of this game with discount factor $\delta$.

Suppose that all players are using strategies of the following form:

$$
\pi_{a_{i}, n_{i}}^{i}=\operatorname{Pr}\{C \mid a\} .
$$

In words, each player bases her own randomizations solely on her own past actions and the number of other players that cooperated, not their identity. The next theorem shows that outcomes arbitrarily close to the efficient outcome can be supported

Theorem 5 For any $v \in(0, N)$ there is a $\bar{\delta}$ such that for all $\delta>\bar{\delta}, G^{\infty}(\delta)$ has a completely mixed SPE in which each player obtains a payoff of $v$.

Proof: Construct the following sequence of numbers:

$$
V(0)=v_{0}, V(n)=v_{0}+\frac{n}{(N-1)}\left(v-v_{0}\right) \text { for } n \in\{1, \ldots, N-1\} .
$$

many periods before reverting to an equilibrium in the set $(-1,0)^{2}$. These strategeis are robust by the argument made in section 3

${ }^{10}$ Obara (1999b) also analyzes the $N$-player prisoner's dilemma. He uses an extension of the Sekiguchi (1997) approach to obtain an approximately efficient symmetric equilibrium. 
We show that each $V(n)$ can be interpreted as the value to a player in the game when $n$ other players cooperate at the current stage.

To simplify notation, write the strategies as:

$$
\pi_{C, n}=1-\gamma_{n} \text { and } \pi_{D, n}=\beta_{n} .
$$

Note that we have dropped the superscript as we will now restrict attention to symmetric strategies. We are interested in the existence of equilibria in strategies of this type for $\delta$ large enough. The dynamic programming equations become then for $n \in\{0, \ldots, N-1\}$ :

$$
\begin{aligned}
& V(n)=(1-\delta) n+\delta E_{D, n} V\left(n^{\prime}\right) \\
& V(n)=(1-\delta)(n-1)+\delta E_{C, n} V\left(n^{\prime}\right),
\end{aligned}
$$

where $E_{C, n} V\left(n^{\prime}\right)$ denotes the expected value from tomorrow on conditional on the action profile today The transition probabilities are nonlinear in $\left(\gamma_{n}, \beta_{n}\right)$ since they are obtained from two binomial distributions.

Suppose that the strategies used by the players depend on $\delta$ as follows:

$$
\left.\gamma_{n}(\delta)\right|_{\delta=1}=0,\left.\beta_{n}(\delta)\right|_{\delta=1}=0 \text { for all } n \in\{0, \ldots, N-1\} \text {. }
$$

In this case, the equations in (13) are trivially satisfied at $\delta=1$, and in particular, the sequence given in 12 solves the system. Write $\gamma(\delta)=$ $\left(\gamma_{0}(\delta), \ldots, \gamma_{N-1}(\delta)\right)$ and $\boldsymbol{\beta}(\delta)=\left(\beta_{0}(\delta), \ldots, \beta_{N-1}(\delta)\right)$. We want to use the implicit function theorem to conclude that the nonlinear system, 13 has a solution $(\boldsymbol{\gamma}(\delta), \boldsymbol{\beta}(\delta))$ for $\delta$ in a neighborhood of 1 . Next, to guarantee that the solutions are indeed probabilities, we need to show that for all $n, \frac{\partial \gamma_{n}}{\partial \delta}(1)>0$ and $\frac{\partial \beta_{n}}{\partial \delta}(1)>0$

To apply the implicit function theorem, we treat (13) as a system of $2 N$ equations that implicitly define the $2 N$ probabilities $(\boldsymbol{\gamma}(\delta), \boldsymbol{\beta}(\delta))$. Write this system as

$$
V(\boldsymbol{\gamma}, \boldsymbol{\beta} ; \delta)=0
$$

with $\boldsymbol{\gamma}, \boldsymbol{\beta}$ as the endogenous variables and $\delta$ as the exogenous variable.

Viewing (13) as a system of $N-1$ pairs of equations, denote by $V_{n D}$ the first function of the $n$th pair and $V_{n C}$ the second. We now evaluate the partial derivatives of the system at the original solution (for $\delta=1$ ) with 
respect to the endogenous variables. For $1 \leq n \leq N-1$,

$$
\begin{aligned}
\frac{\partial V_{n D}}{\partial \gamma_{n-1}} & =\frac{\partial V_{n C}}{\partial \gamma_{n}}=n(V(n-1)-V(n)) \\
\frac{\partial V_{n D}}{\partial \beta_{n}} & =\frac{\partial V_{n C}}{\partial \beta_{n+1}}=(N-n-1)(V(n+1)-V(n))
\end{aligned}
$$

It can easily be verified that this system has full rank. The implicit function theorem then guarantees the existence of a differentiable solution mapping $(\boldsymbol{\gamma}(\delta), \boldsymbol{\beta}(\delta)$ in the neighborhood of $\delta=1$. To check the sign of the derivatives, we apply the chain rule. Note

$$
D_{\delta} V(\boldsymbol{\gamma}, \boldsymbol{\beta} ; \delta)=\left(\begin{array}{c}
V(0) \\
V(0)-1 \\
V(1)-1 \\
V(1)-2 \\
\vdots \\
V(N-1)-(N-1) \\
V(N-1)-N
\end{array}\right)
$$

Observe that the system of equations,

$$
D_{\boldsymbol{\gamma}, \boldsymbol{\beta}} V(\boldsymbol{\gamma}, \boldsymbol{\beta} ; \delta) D_{\delta}(\boldsymbol{\gamma}(\delta), \boldsymbol{\beta}(\delta))=-D_{\delta} V(\boldsymbol{\gamma}, \boldsymbol{\beta} ; \delta)
$$

can be solved in blocks of size 2 . This makes the comparative statics relatively easy:

$$
\begin{aligned}
& \left(\begin{array}{cc}
n(V(n-1)-V(n)) & (N-n-1)(V(n+1)-V(n)) \\
(n+1)(V(n)-(V(n+1))) & (N-n-2)(V(n+2)-V(n+1))
\end{array}\right)\left(\begin{array}{c}
\frac{\partial \gamma_{n}}{\partial \delta} \\
\frac{\partial \beta_{n}}{\partial \delta}
\end{array}\right) \\
& =-\left(\begin{array}{c}
V(n)-n+1 \\
V(n+1)-n-1
\end{array}\right) \text {. }
\end{aligned}
$$

Using the fact that $V(n+1)-V(n)=V(k)-V(k-1)$ for all $k, n \in$ $\{0,1, \ldots, N-1\}$, it is easy to see that the derivatives have the right sign whenever $v_{0}$ is chosen close enough to $v$. Hence for $\delta$ close enough to one, the system can be solved for probabilities. This implies that for such discount factors, the game has a completely mixed SPE with a payoff $v$. 
In order to obtain the result on games with almost perfect monitoring, fix a $\delta$ for which there is a completely mixed SPE with payoff vector $v$. We can make the same continuity argument as in the previous section to conclude that whenever the monitoring is close enough to perfect, a sequential equilibrium with payoff vector $v$ exists.

\section{Conclusion}

There are many unresolved questions in the context of discounted repeated games with private monitoring. In particular, unlike the public monitoring case, there is no known folk theorem for fixed monitoring technologies. ${ }^{11}$ In this note, we have followed the literature and sought after the weaker result: a limit folk theorem for nearly perfect monitoring. An important goal for future research is to characterize equilibrium payoffs when monitoring is private but far from perfect. The strategies we have used in this paper can sustain some cooperation under less than perfect monitoring technologies and equilibrium can be characterized by an analogous system of dynamic programming equations. The payoffs that can be supported are thus those values for which the system can be solved for probabilities. Determining the full potential of this approach is ongoing research.

\section{References}

Abreu, D., D. Pearce, and E. Stachetti (1986): "Optimal Cartel Monitoring with Imperfect Information," Journal of Economic Theory, 39, 251-269.

(1990): "Toward a Theory of Discounted Repeated Games with Imperfect Monitoring," Econometrica, 58, 1041-1063.

Bhaskar, V. (1998): "Informational Constraints and the Overlapping Generations Model: Folk and Anti-Folk Theorems," Review of Economics Studies, 65, 135-149.

(1999): "Sequential Equilibria in the Repeated Prisoners' Dilemma with Private Monitoring," mimeo, University of Essex.

\footnotetext{
${ }^{11}$ Lehrer (1989) and Lehrer (1990) prove folk theorems for non-discounting criteria
} 
FudenberG, D., D. Levine, And E. Maskin (1994): "The Folk Theorem with Imperfect Public Information," Econometrica, 62(5), 997-1040.

FudenberG, D., And E. Maskin (1986): "The Folk Theorem in Repeated Games with Discounting and with Incomplete Information," Econometrica, 54, 533-554.

(1991): "On the Dispensability of Public Randomization in Discounted Repeated Games," Journal of Economic Theory, 53, 428-438.

Green, E. J., And R. H. Porter (1984): "Noncooperative Collusion under Imperfect Price Information," Econometrica, 52(1), 87-100.

Lehrer, E. (1989): "Lower Equilibrium Payoffs in Two-Player Repeated Gaes with Non-Observable Actions," International Journal of Game Theory, 18(1), 57-89.

(1990): "Nash Equilibrium of n-Player Repeated Games with SemiStandard Information," International Journal of Game Theory, 19, 191217.

Mailath, G. J., And S. Morris (1998): "Repeated Games with Imperfect Private Monitoring: Notes on a Coordination Perspective," CARESS Working Paper 98-07, University of Pennsylvania.

Matsushima, H. (1991): "On the Theory of Repeated Games with Private Information, part I: Anti-Folk Theorem without Communication," Economics Letters, 35, 253-256.

ObarA, I. (1999a): "Private Strategy and Efficiency: Repeated Partnership Game Revisited," mimeo, University of Pennsylvania.

- (1999b): "The Repeated Prisoner's Dilemma with Private Monitoring: a N-player Case," CARESS Working Paper \#99-13, University of Pennsylvania.

Piccione, M. (1998): “The Repeated Prisoners' Dilemma with Imperfect Private Monitoring," mimeo.

RADNER, R. (1985): "Repeated Principal-Agent Games with Discounting," Econometrica, 53(5), 1173-1198. 
Sekiguchi, T. (1997): "Efficiency in Repeated Prisoners' Dilemma with Private Monitoring," Journal of Economic Theory, 76(2), 345-361.

Shin, H. S., and T. Williamson (1991): "How Much Common Belief is Necessary for a Convention?," Games and Economic Behavior, 13, 252268. 